\title{
Elastic constants of hard thin platelets by Monte Carlo simulation and virial expansion
}

\author{
Paul A. O’Brien, Michael P. Allen, and David L. Cheung \\ Department of Physics and Centre for Scientific Computing, University of Warwick, Coventry CV4 7AL, United Kingdom \\ Matthew Dennison and Andrew Masters \\ School of Chemical Engineering and Analytical Science, University of Manchester, Sackville Street, Manchester M60 1QD, \\ United Kingdom
}

(Received 2 September 2008; published 24 November 2008)

\begin{abstract}
In this paper we present an investigation into the calculation of the Frank elastic constants of hard platelets via molecular simulation and virial expansion beyond second order. Monte Carlo simulations were carried out and director fluctuations measured as a function of wave vector $\boldsymbol{k}$, giving the elastic constants through a fit in the low- $k$ limit. Additionally, the virial expansion coefficients of the elastic constants up to sixth order were calculated, and the validity of the theory determined by comparison with the simulation results. The simulation results are also compared with experimental measurements on colloidal suspensions of platelike particles.
\end{abstract}

DOI: 10.1103/PhysRevE.78.051705

PACS number(s): 61.30.Cz, 61.20.Ja, 05.20.-y

\section{INTRODUCTION}

The liquid crystalline nematic phase, characterized by long-range orientational order and translational disorder of the molecules, is typically found in systems of rodlike and platelike molecules [1]. Indeed, since the theoretical work of Onsager [2] and the simulations of Frenkel and co-workers [3-6], it has been known that entropic effects alone, which result from the excluded volumes of sufficiently aspherical particles, are sufficient to drive the isotropic-nematic transition. Therefore, hard-particle models capture some of the essential physics, and have been used extensively in computer simulations. The results of such simulations may be compared, quantitatively, with experimental studies of colloidal suspensions of rodlike and platelike solid particles [7-9].

Within the nematic phase, molecular orientations are distributed about a preferred direction: The so-called director $\boldsymbol{n}$. Spatial deformations of the director are well described, in the limit of large wavelength, by the Oseen-Frank $[10,11]$ orientational elastic theory, which contains three phenomenological parameters, the Frank elastic constants. Experimentally, knowledge of these constants, together with a few other parameters (such as surface anchoring strengths), is sufficient to describe many of the interesting large-scale and mesoscale structures formed in liquid crystals.

For rodlike particles, computer simulations were used long ago to relate Frank elastic constants to molecular shape [12-15]. Onsager's theory, which amounts to a classical density-functional description of the distortion free energy at the second virial coefficient level (neglecting higher-thanpairwise interactions) gives an accurate description of both the thermodynamics and orientational elastic properties of sufficiently elongated rods [16], and can be empirically modified to give reasonable accuracy at moderate elongations $[14,17,18]$. The reason for this is well understood: The reduced higher virial coefficients $B_{n} / B_{2}^{(n-1)}$ are known to vanish as the molecular elongation tends to infinity $[19,20]$. Calculations of elastic constants from molecular dynamics simulation have also been performed for Gay-Berne disks [21], and recent experiments examining the effects of exter- nal fields and interfaces on the nematic phase of platelike colloids [22-24] also shed light on the values of the Frank constants, and invite comparison with computer simulations and theory. However, for oblate molecules (even infinitesimally thin plates), it is known that the higher virial coefficients are significant, and Onsager theory gives a poor description of the phase behavior [3,4]. Approximate resummations of the virial series, such as the Parsons-Lee approach $[25,26]$, will fail in the infinitely thin plate limit, as the volume of the particle is zero.

This paper presents Monte Carlo calculations of the Frank elastic constants for systems composed of thin hard circular disks over their full nematic range, and compares with theoretical estimates which incorporate virial terms up to sixth order. The paper is arranged as follows. Section II reviews the orientational elastic theory of Frank and Oseen, and describes the fitting procedures used to extract the elastic constants from simulation. Section III summarizes the virial theory for calculating the elastic constants. Section IV gives the simulation details. The results are presented in Sec. V and conclusions in Sec. VI.

\section{ELASTIC THEORY}

For bulk systems in the nematic phase, slowly varying spatial inhomogeneities in the director field $\boldsymbol{n}$ give rise to the following free energy penalty [11]:

$$
\begin{aligned}
\Delta \mathcal{F}= & \frac{1}{2} \int d \boldsymbol{r}\left\{K_{1}[\boldsymbol{\nabla} \cdot \boldsymbol{n}(\boldsymbol{r})]^{2}+K_{2}[\boldsymbol{n}(\boldsymbol{r}) \cdot[\boldsymbol{\nabla} \wedge \boldsymbol{n}(\boldsymbol{r})]]^{2}\right. \\
& \left.+K_{3}|\boldsymbol{n}(\boldsymbol{r}) \wedge[\boldsymbol{\nabla} \wedge \boldsymbol{n}(\boldsymbol{r})]|^{2}\right\},
\end{aligned}
$$

where $K_{1}, K_{2}$, and $K_{3}$ are the splay, twist, and bend Frank elastic constants, respectively. The rotational invariance of bulk geometry means that the average director $\boldsymbol{n}$ can vary with time throughout simulations, leading to complications in describing system quantities. For this treatment we require a consistent director, hence we consider also the effect of a small orienting field giving the following contribution to the free energy: 


$$
\mathcal{F}_{\text {ext }}=\Phi \int d \boldsymbol{r}\left\{1-[\boldsymbol{n}(\boldsymbol{r}) \cdot \boldsymbol{z}]^{2}\right\}
$$

where $z$ is the unit vector in the $z$ direction, and $\Phi$ is a strength parameter. This can be chosen to be reasonably small while preventing $\boldsymbol{n}$ from wandering far from $\boldsymbol{z}$.

The thermal fluctuations of the director are best described by a spectrum of modes. Decomposing the director with the Fourier transform pair

$$
\begin{gathered}
\boldsymbol{n}(\boldsymbol{r})=\frac{1}{V} \sum_{\boldsymbol{k}} \widetilde{\boldsymbol{n}}(\boldsymbol{k}) \exp (-i \boldsymbol{k} \cdot \boldsymbol{r}), \\
\widetilde{\boldsymbol{n}}(\boldsymbol{k})=\int \boldsymbol{n}(\boldsymbol{r}) \exp (i \boldsymbol{k} \cdot \boldsymbol{r}) d \boldsymbol{r}
\end{gathered}
$$

allows one to work in reciprocal space with the Fourier components $\widetilde{\boldsymbol{n}}(\boldsymbol{k})$. A consistent manner of describing such spectra involves a change of coordinate system from the fixed $\boldsymbol{x y}$. frame to a $\boldsymbol{k}$-dependent $\mathbf{1 2 3}$ frame, where $\mathbf{3}$ is fixed along the desired director (here the $z$ axis), $\mathbf{1}$ is defined perpendicular to $\mathbf{3}$ such that the wave vector $\boldsymbol{k}$ is in the $\mathbf{1 3}$ plane and $\mathbf{2}$ is perpendicular to both $\boldsymbol{n}$ and $\boldsymbol{k}$. Hence, the wave-vector coordinates reduce to $\boldsymbol{k}=\left(k_{1}, 0, k_{3}\right)$.

For small variations of $\boldsymbol{n}$ away from the 3-direction, $n_{3}(\boldsymbol{r}) \approx 1$, and the distortion free energy, perturbed by the external field, can be written in terms of the Fourier transforms $\tilde{n}_{1}(\boldsymbol{k}), \tilde{n}_{2}(\boldsymbol{k})$, of the remaining components,

$$
\begin{aligned}
\mathcal{F}= & \frac{1}{2 V} \sum_{\boldsymbol{k}} K_{1} k_{1}^{2}\left|\widetilde{n}_{1}(\boldsymbol{k})\right|^{2}+K_{2} k_{1}^{2}\left|\widetilde{n}_{2}(\boldsymbol{k})\right|^{2} \\
& +\left(K_{3} k_{3}^{2}+2 \Phi\right)\left[\left|\widetilde{n}_{1}(\boldsymbol{k})\right|^{2}+\left|\widetilde{n}_{2}(\boldsymbol{k})\right|^{2}\right] .
\end{aligned}
$$

According to equipartition of energy, each mode is assigned an average energy of $k_{B} T / 2$ and hence

$$
\left\langle\left|\tilde{n}_{\mu}(\boldsymbol{k})\right|^{2}\right\rangle=\frac{V k_{B} T}{K_{\mu} k_{1}^{2}+K_{3} k_{3}^{2}+2 \Phi}, \quad \mu=1,2 .
$$

This is valid in the limit of low $k$.

\section{VIRIAL THEORY}

The Helmholtz energy, $F$, of a system with number density $\rho=N / V$, and temperature $T$ is given by

$$
\begin{aligned}
F= & F_{0}+N k_{B} T\left(\ln \left(\Lambda^{3} \rho\right)-1+\int \varphi(\boldsymbol{u}) \ln [4 \pi \varphi(\boldsymbol{u})] d \boldsymbol{u}\right. \\
& \left.+\sum_{n=2} \frac{1}{n-1} B_{n} \rho^{n-1}\right) .
\end{aligned}
$$

$F_{0}$ is the ideal gas contribution, related to rotational degrees of freedom, $B_{n}$ is the $n$th virial coefficient, $\Lambda$ is the thermal de Broglie wavelength, and $\varphi(\boldsymbol{u})$ is the one-particle distribution function of particle orientation $\boldsymbol{u} . B_{n}$ is given by

$$
B_{n}=\frac{1-n}{n ! V} \int \cdots \int \varphi\left(\boldsymbol{u}_{1}\right) \cdots \varphi\left(\boldsymbol{u}_{n}\right) V_{n} d \boldsymbol{u}_{1} \cdots d \boldsymbol{u}_{n} d \boldsymbol{r}_{1} \cdots d \boldsymbol{r}_{n},
$$

where

$$
V_{n}=\sum_{S_{n}} \prod_{i<j}^{n} f_{i j}
$$

The sum over $S_{n}$ denotes the sum over all star integrals with $n$ points, and $f_{i j}$ is the Mayer $f$-bond between particles $i$ and $j$. As we are dealing with hard particles, this is equal to -1 when the particles are overlapping, and 0 when they are not. $\varphi(\boldsymbol{u})$ is determined as the function that minimizes $F$ subject to the normalization condition

$$
\int \varphi(\boldsymbol{u}) d \boldsymbol{u}=1
$$

At low density, the only solution is $\varphi(\boldsymbol{u})=1 / 4 \pi$ (for axially symmetric particles), corresponding to the isotropic phase. At higher densities a nematic solution also appears. The isotropic-nematic coexistence densities are determined by equating the chemical potentials and pressures of the two phases. The calculation of the nematic virial coefficients is done by making use of Onsager's trial function for $\varphi(\boldsymbol{u})[2]$,

$$
\varphi(\boldsymbol{u})=\frac{\alpha}{4 \pi \sinh \alpha} \cosh (\alpha \cos \theta),
$$

where $\alpha$ is a parameter describing the nematic ordering of the system about the director $\boldsymbol{n}$, and ranges from 0 for isotropic ordering (no order), to $\infty$ for perfect nematic ordering. $\theta$ is the angle that each particle makes with this director. In this case, the nematic order parameter, $S=\left\langle P_{2}(\cos \theta)\right\rangle$, where $P_{2}$ is the second Legendre polynomial, is given by

$$
S=1+\frac{3}{\alpha^{2}}-\frac{3}{\alpha \tanh \alpha} .
$$

Using this trial function gives $F$ as a function of $\alpha$, akin to a Landau Helmholtz energy expression. $\alpha$ can then be determined for a given density by requiring $\partial F / \partial \alpha=0$, to give the $\alpha$ value for which $F$ is minimized. Further discussion of the quality of this approximation will be given in a future presentation.

The virial coefficients are calculated using a modified version of the Ree-Hoover method [27,28]. The main difference is that the virial coefficients are calculated for a variety of $\alpha$ values. For a given value of $\alpha$, the particle orientations are chosen at random from the trial function distribution. Repeating this for a range of $\alpha$ values gives the virial as a function of $\alpha$. A cubic spline interpolation is then used to join the discrete points calculated. With this done, it is then possible to minimize $F$ with respect to $\alpha$, and calculate the pressure and chemical potential of the system as a function of density. Detailed results from this method will be given in a later presentation. Suffice it to say that the equation of state of the nematic phase converges rapidly with increasing order of expansion, with very small oscillations about the simulation result. 
The Frank elastic constants of a nematic liquid crystal are calculated in a related manner to the calculation of the equation of state. $K_{1}, K_{2}$, and $K_{3}$ are given by $[29,30]$

$$
\begin{aligned}
K_{\mu}= & \frac{1}{2} \rho k_{B} T \int \cdots \int r_{\mu}^{2} c(1,2) \varphi^{\prime}\left(\boldsymbol{u}_{1}\right) \varphi^{\prime}\left(\boldsymbol{u}_{2}\right) u_{1, x} u_{2, x} \\
& \times d \boldsymbol{u}_{1} d \boldsymbol{u}_{2} d \boldsymbol{r}_{1} d \boldsymbol{r}_{2} .
\end{aligned}
$$

$c(1,2)$ is the two-particle direct correlation function, while $r_{\mu}, \mu=1,2,3$ are the distances between two particles in the $x$, $y$, and $z$ directions, respectively. $\varphi^{\prime}(\boldsymbol{u})$ is the derivative of $\varphi(\boldsymbol{u})$ with respect to $\boldsymbol{u} \cdot \boldsymbol{n}$ (i.e., $\cos \theta$ ). The other symbols have their usual meanings. To solve this integral, an expression for $c(1,2)$ is required. This can be expressed in terms of the Mayer function $f_{i j}$,

$$
c(1,2)=f_{12}+\rho \int f_{12} f_{23} f_{13} \varphi\left(\boldsymbol{u}_{3}\right) d \boldsymbol{r}_{3} d \boldsymbol{u}_{3}+\cdots .
$$

The expansion takes into account the Mayer function between each pair of particles at each virial level. Using this expansion, the integral can be split into components at each virial level. The general expression for each coefficient at virial level $B_{n}$ is

$$
\begin{aligned}
K_{\mu}^{(n)}= & \frac{1-n}{n ! V} \int \cdots \int V_{n} r_{\mu}^{2} \varphi^{\prime}\left(\boldsymbol{u}_{1}\right) \varphi^{\prime}\left(\boldsymbol{u}_{2}\right) \varphi\left(\boldsymbol{u}_{3}\right) \cdots \\
& \times \varphi\left(\boldsymbol{u}_{n}\right) u_{1, x} u_{2, x} d \boldsymbol{u}_{1} \cdots d \boldsymbol{u}_{n} d \boldsymbol{r}_{1} \cdots d \boldsymbol{r}_{n},
\end{aligned}
$$

where $V_{n}$ is given by Eq. (9). The coefficients are then summed to give the elastic constants

$$
K_{\mu}=k_{B} T \sum_{n=2} \frac{K_{\mu}^{(n)} \rho^{n}}{n-1} .
$$

Again, a modified Ree-Hoover method [27,28] is used to calculate the coefficients for any given value of $\alpha$. To compare with simulation, we can either take the order parameter $S$ from simulation and calculate the corresponding $\alpha$, or we can use the $\alpha$ calculated by the theoretical equation of state.

\section{SIMULATION DETAILS}

The simulated system consists of a fluid of infinitely thin hard disks (interaction potential between a pair of molecules $V_{i j}=\infty$ if the molecules overlap, and zero otherwise). The overlap criterion is described elsewhere [4,31]. For this system, due to the absence of any finite interaction potentials, the temperature is irrelevant, except as a scaling factor for energies and free energies. Reduced units of energy in these simulations are obtained by taking $k_{B} T=1$ and reduced units of distance by setting the platelet diameter $D=1$.

The orienting field of Eq. (2) is applied in the simulation as the following potential energy term:

$$
\mathcal{U}_{\mathrm{ext}}=U \sum_{i=1}^{N}\left[1-\left(\boldsymbol{u}_{i} \cdot \boldsymbol{z}\right)^{2}\right]
$$

where $\boldsymbol{u}_{i}$ denotes the orientation of molecule $i$ and $U$ is a field strength parameter. It is easy to show that the strength
TABLE I. Density $\rho D^{3}$ of each simulation, with simulation averages of order parameter $S$, and elastic constants $K_{\mu} D / k_{B} T$. Numbers in parentheses represent estimated errors in the last quoted digit.

\begin{tabular}{lcccc}
\hline \hline$\rho D^{3}$ & $S$ & $K_{1} D / k_{B} T$ & $K_{2} D / k_{B} T$ & $K_{3} D / k_{B} T$ \\
\hline 4.50 & $0.6694(8)$ & $2.85(4)$ & $4.75(7)$ & $0.86(1)$ \\
5.00 & $0.7620(4)$ & $4.3(3)$ & $7.6(1)$ & $0.98(4)$ \\
6.00 & $0.8490(2)$ & $9.80(5)$ & $15.2(1)$ & $1.31(3)$ \\
6.25 & $0.8618(3)$ & $11.4(1)$ & $17.4(1)$ & $1.43(4)$ \\
6.50 & $0.8738(3)$ & $13.4(1)$ & $20.6(3)$ & $1.63(4)$ \\
6.75 & $0.8827(3)$ & $14.9(1)$ & $22.8(1)$ & $1.56(9)$ \\
7.00 & $0.8922(2)$ & $17.4(3)$ & $26.2(4)$ & $1.59(1)$ \\
7.25 & $0.9003(3)$ & $19.3(2)$ & $28.9(2)$ & $1.75(6)$ \\
7.50 & $0.9065(2)$ & $21.5(1)$ & $32.8(3)$ & $1.76(4)$ \\
7.75 & $0.9130(2)$ & $24.2(1)$ & $36.1(5)$ & $1.80(3)$ \\
8.00 & $0.9180(3)$ & $26.7(3)$ & $40.5(2)$ & $1.89(5)$ \\
\hline \hline
\end{tabular}

parameter of Eq. (2) is related to $U$ by $\Phi=\rho S U$. A value $U / k_{B} T=0.1$ was found to ensure the $z$ component of $\boldsymbol{n}$ remained within $0.1 \%$ of 1 .

Monte Carlo simulations were performed for systems of 8000 particles with cubic periodic boundary conditions in the canonical ensemble. Equilibration runs consisted of at least $10^{5}$ sweeps, and simulation averages were taken during production runs of 1 or $2 \times 10^{6}$ sweeps, depending on density (1 sweep equals 1 attempted MC move, combined translation and rotation, per particle). Displacement parameters were chosen to give a move acceptance rate in the range $30 \%-$ $40 \%$. All of the state points chosen, with a density range $\rho D^{3}=4.5-8$, are in the nematic region for platelets; the isotropic-nematic transition occurs at $\rho D^{3} \approx 3.78[4,32]$. Average values of order parameter for each density are reported in Table I.

The use of Eq. (5) to calculate elastic constants from molecular simulations is well established [12-15], and so only a brief description will be given here. It is convenient to work with the order tensor, $Q_{\mu \nu}$, for the representation of orientational correlations in the nematic phase. For molecular orientations represented by unit vectors $\boldsymbol{u}_{i}=\left(u_{i x}, u_{i y}, u_{i z}\right)$,

$$
Q_{\mu \nu}(\boldsymbol{r})=\frac{V}{N} \sum_{i=1}^{N} \frac{3}{2}\left(u_{i \mu} u_{i \nu}-\frac{1}{3} \delta_{\mu \nu}\right) \delta\left(\boldsymbol{r}-\boldsymbol{r}_{i}\right),
$$

where $\mu, \nu=x, y, z, \delta_{\mu \nu}$ is the Kronecker $\delta$ and $\delta(\cdots)$ the Dirac $\delta$ function. The order parameter $S$, an indication of the degree of mutual alignment, is found from the highest eigenvalue of the volume-averaged order tensor, with the director $\boldsymbol{n}$ given by the corresponding eigenvector. It is easy to show that $S=\left\langle P_{2}\left(\boldsymbol{u}_{i} \cdot \boldsymbol{n}\right)\right\rangle$ as defined earlier. The Fourier components of the order tensor,

$$
\tilde{Q}_{\mu \nu}(\boldsymbol{k})=\frac{V}{N} \sum_{i=1}^{N} \frac{3}{2}\left(u_{i \mu} u_{i \nu}-\frac{1}{3} \delta_{\mu \nu}\right) \exp \left(i \boldsymbol{k} \cdot \boldsymbol{r}_{i}\right),
$$

can be related, at low $k$, to those of the director via the identity 


$$
\left\langle\left|\widetilde{Q}_{\mu 3}(\boldsymbol{k})\right|^{2}\right\rangle \equiv \frac{9}{4} S^{2}\left\langle\left|\widetilde{n}_{\mu}(\boldsymbol{k})\right|^{2}\right\rangle
$$

and thus we obtain for the order tensor spectrum [33]

$$
\left\langle\left|\tilde{Q}_{\mu 3}(\boldsymbol{k})\right|^{2}\right\rangle=\frac{\frac{9}{4} S^{2} V k_{B} T}{K_{\mu} k_{\mu}^{2}+K_{3} k_{3}^{2}+2 \rho S U} .
$$

The elastic constants can be obtained from the function

$$
\mathcal{W}_{\mu 3}(\boldsymbol{k}) \equiv \frac{\frac{9}{4} S^{2} V k_{B} T}{\left\langle\left|\widetilde{Q}_{\mu 3}(\boldsymbol{k})\right|^{2}\right\rangle} \rightarrow K_{\mu} k_{\mu}^{2}+K_{3} k_{3}^{2}+2 \rho S U \quad \text { as } k \rightarrow 0,
$$

fitted to the simulation data. In the case of high $k$ the function will converge to a limiting value

$$
\mathcal{W}_{\mu 3}(k) \rightarrow \frac{\left\langle P_{2}^{2}\right\rangle \rho k_{B} T}{\frac{1}{21}\left\langle P_{2}\right\rangle-\frac{4}{35}\left\langle P_{4}\right\rangle+\frac{1}{15}} \quad \text { as } k \rightarrow \infty,
$$

involving both average second- and fourth-order polynomials of $\boldsymbol{u}_{i} \cdot \boldsymbol{n}$.

In the simulation, the wave vectors of orientational distortions are determined by the size of the system via

$$
\boldsymbol{k}=k_{0}\left(\kappa_{x}, \kappa_{y}, \kappa_{z}\right) \text {, }
$$

where $k_{0}=2 \pi / L, L$ is the length of the simulation box and the $\kappa_{\mu}$ are integers chosen in the range $0 \leqslant \kappa_{x, y} \leqslant 20$ and $0 \leqslant \kappa_{z} \leqslant 40$, the upper limit being large enough to observe convergence of $\mathcal{W}_{\mu 3}$ to the high- $k$ limit. The calculation of director fluctuation spectra is quite expensive, and the low- $k$ fluctuations are quite slow: Therefore, contributions to $\mathcal{W}_{\mu 3}$ were computed every $100 \mathrm{MC}$ sweeps for low values of $k$ $\left(\kappa_{x, y}<10\right.$ and $\left.\kappa_{z}<20\right)$, and every 1000 sweeps for the higher- $k$ modes which are less critical in estimating the elastic constants. Other quantities, such as pressure and order tensor, were calculated every 10 sweeps. In order to obtain the director fluctuations in a convenient form, the molecular orientations must first be transformed from the fixed $\boldsymbol{x y}$. frame into the $\boldsymbol{k}$-dependent $\mathbf{1 2 3}$ coordinates system. The functions $\left|\widetilde{Q}_{\mu 3}(\boldsymbol{k})\right|^{2}$ can then be calculated for the desired wave-vector spectrum from Eq. (18), saved at regular intervals and statistical uncertainties estimated from the variation over several blocks of MC sweeps.

After obtaining the spectra, the functions $\mathcal{W}_{\mu 3}(\boldsymbol{k})$ were found from Eq. (21) and were fitted to a ratio of multivariate polynomials in $k_{1}^{2}$ and $k_{3}^{2}$. The polynomials were chosen so as to ensure that the leading coefficients in the numerator provided estimates for the elastic constants and the trial function converged to the limit of Eq. (22) for high $k$.

In the least-squares fitting of the trial function to fluctuation data, the estimates for the elastic constants are sensitive to several details of the fitting procedure. The range of the $\boldsymbol{k}$ spectrum used in the fit largely determines the extent to which the fitting function describes the behavior at high $k$, whereas an accurate description of the low- $k$ modes will give the most accurate values for the elastic constants. Hence, changing the limiting values of $k_{1}$ and $k_{3}$ will change the relative weighting of the low- $k$ data in the fit. Another feature of the fitting is the number of parameters in the fitting function, determining the freedom of the function to match the

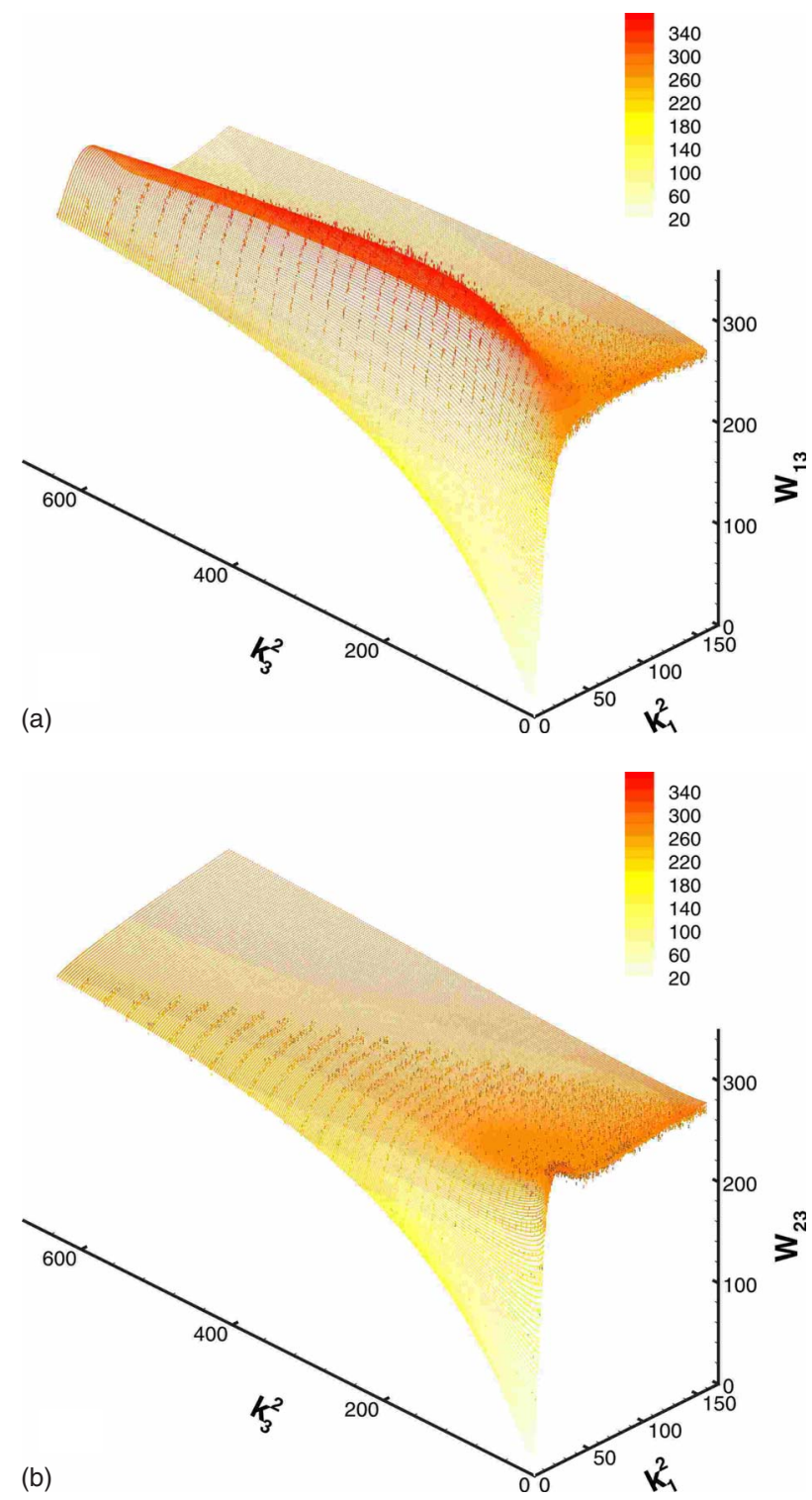

FIG. 1. (Color online) The functions (a) $\mathcal{W}_{13}$, and (b) $\mathcal{W}_{23}$, as functions of squared wave-vector components $\left(k_{1}^{2}, k_{3}^{2}\right)$ at the $\rho D^{3}$ $=8$ state point. The simulation data with statistical error is given as a set of vertical lines, with the dotted surface representing the fitting function. The legends show the contour color coding, with all units such that $D=1$ and $k_{B} T=1$.

simulation data. Increasing the number of parameters can allow the function to reduce the accuracy for the low- $k$ data in favor of following more closely for higher $k$. Several choices for the number of parameters and range of $\boldsymbol{k}$ spectrum were used, and the leading coefficients averaged over these choices to provide estimates for the elastic constants with associated fitting error. These limits imposed by the fitting turned out to be larger than the statistical errors for the runs conducted here.

\section{RESULTS}

Figure 1 shows the functions $\mathcal{W}_{\mu 3}(\boldsymbol{k})$, defined by Eq. (21). As desired, the fitting functions describe the linear, low- $k$ 

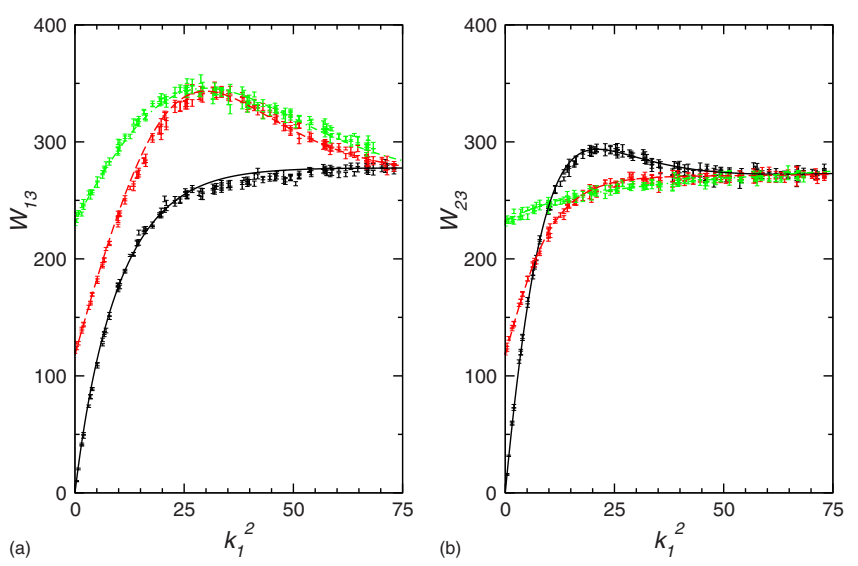

FIG. 2. (Color online) Slices through the surfaces of (a) $\mathcal{W}_{13}$, and (b) $\mathcal{W}_{23}$, for fixed $k_{3}$ as a function of $k_{1}^{2}$, showing respectively splay and twist fluctuations, from simulations at the $\rho D^{3}=8$ state point. The error bars give the data points with statistical error and the lines correspond to the fitting function for the entire surface in each case. $k_{3}=0$, full line (black); $k_{3}=15 k_{0}$, dashed line (red); $k_{3}$ $=30 k_{0}$, dot-dashed line (green).

regime well, and even pass through most of the data points for higher $k . \mathcal{W}_{13}$ follows the structure over a maximum as $k_{1}$ and $k_{3}$ increase together and $\mathcal{W}_{23}$ describes the smoothing out of a sharply peaked profile in $k_{1}$ as $k_{3}$ increases. It is apparent that the low bend elastic constant, causing only gradually increasing fluctuations, couples with a lack of structure developing along the director (compared to normal fluctuations) to allow more points to be included in the low- $k$ treatment along $\boldsymbol{n}$.

This behavior is also illustrated in Figs. 2 and 3, showing various slices of the data sets for $\mathcal{W}_{\mu 3}(\boldsymbol{k})$. Although the estimates for the elastic constants are sensitive to the range of wave-vector spectrum and number of fitting parameters
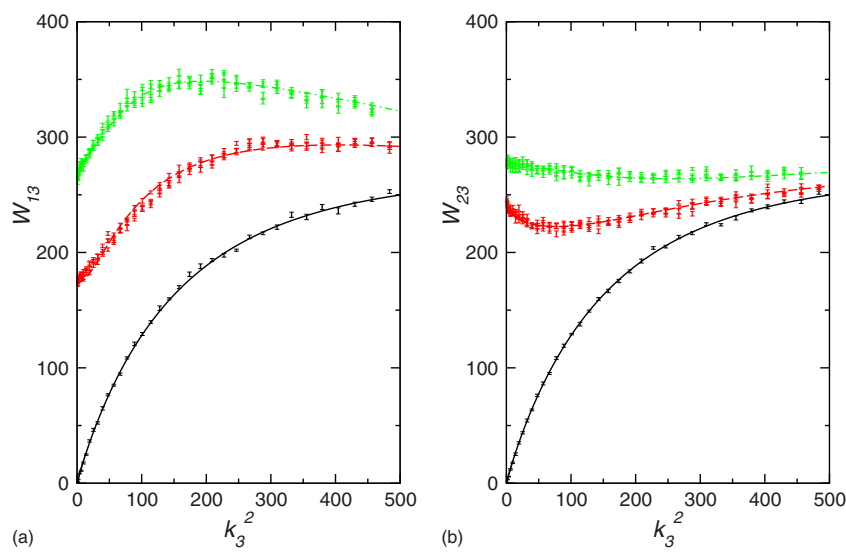

FIG. 3. (Color online) Slices through the surfaces of (a) $\mathcal{W}_{13}$ and (b) $\mathcal{W}_{23}$ for fixed $k_{1}$ as a function of $k_{3}^{2}$, showing bend fluctuations, from simulations at the $\rho D^{3}=8$ state point. The error bars give the data points with statistical error and the lines correspond to the fitting function for the entire surface in each case. $k_{1}=0$, full line (black); $k_{1}=5 k_{0}$, dashed line (red); $k_{1}=10 k_{0}$, dotted-dashed line (green).

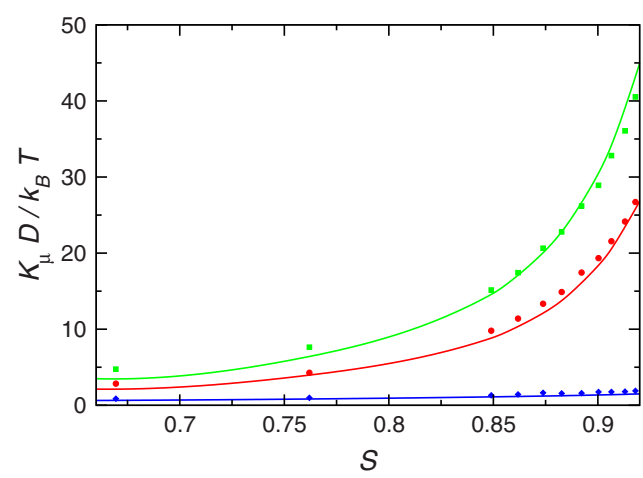

FIG. 4. (Color online). The splay, twist, and bend elastic constants as a function of order parameter. The lines represent data from a sixth-order virial expansion, and the points are simulation results. Splay, $K_{1}$, circles (red); twist, $K_{2}$, squares (green); bend, $K_{3}$, diamonds (blue). In all cases the errors are smaller than the plotting symbols.

included, there is no visible effect on the low- $k$ regions of each graph. Hence, we have plotted the fitting function as fitted across the entire spectrum with the largest number of parameters used, in order to guide the eye over the observed data.

The elastic constants obtained from this fitting procedure are summarized in Table I. Figure 4 shows the elastic constants as a function of order parameter as obtained from the simulation and theory. The qualitative features of the results are as expected for disklike particles [34]: Director twist induces the largest free energy penalty, bend is the least expensive, and splay lies between the others. A striking result is that the bend elastic constant $K_{3}$ is very small compared with the others (by more than an order of magnitude for the more ordered state points); this highlights the anisotropy of the infinitely thin platelets.

It is of interest to compare these results with experimental determinations of the elastic constants for colloidal suspensions of gibbsite particles [23,24]: $K_{1}=0.6-2.6 \times 10^{-13} \mathrm{~N}$ and $K_{3}=6-8 \times 10^{-14} \mathrm{~N}$ for an order parameter in the range $S \approx 0.80-0.85$. If one inserts estimates for $D \approx 220 \mathrm{~nm}$ and $k_{B} T \approx 4 \times 10^{-21}$, the corresponding simulation results for $S \approx 0.85$ are $K_{1} \approx 1.8 \times 10^{-13} \mathrm{~N}$ and $K_{3} \approx 2.4 \times 10^{-14} \mathrm{~N}$. This is quite reasonable agreement, bearing in mind that the experimental platelets are not of vanishing thickness.

The virial coefficients for the Frank elastic constants have been calculated up to sixth order. At higher orders, statistical errors were too large to give acceptable reults. This is due to the presence of $\varphi^{\prime}(\boldsymbol{u})$ in the integrand given by Eq. (14), which varies sharply for the more ordered distributions. We use the order parameter determined by simulation at a given density to fix the value of $\alpha$ [Eq. (11)], calculate the elastic constant virial series at this particular density, and then study its convergence properties.

Figure 4 compares results from the virial expansion theory with simulation data, showing reasonable agreement. Figure 5 shows the convergence of the virial series for the splay constant $K_{1}$. As can be seen, truncating at the secondorder virial level drastically underestimates the elastic constant. However, at third-order virial level, the agreement with 


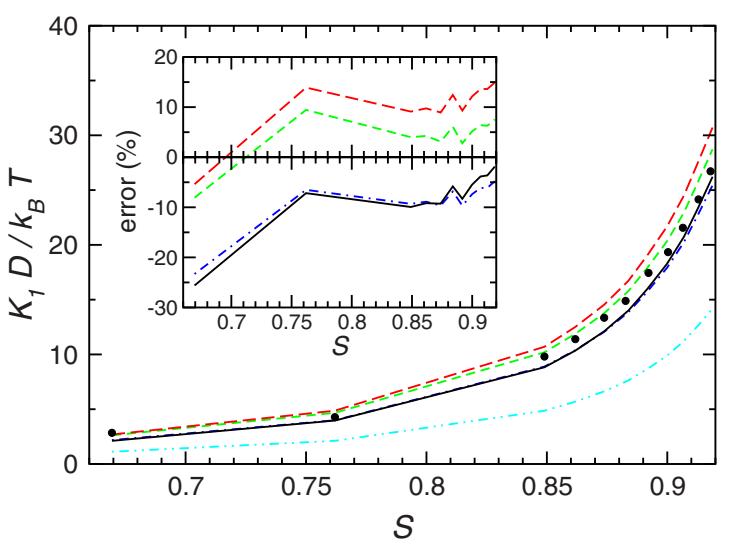

FIG. 5. (Color online) Splay elastic constant $K_{1}$ as a function of order parameter $S$. The black circles give results from Monte Carlo simulation, the lines give data from various orders of virial expansion. Sixth order, continuous (black) line; fifth order, long dashed (red) line; fourth order, short dashed (green) line; third order, dotted-dashed (blue) line; second order, double-dotted-dashed (cyan) line. Inset: Percentage error in the third- through sixth-order theories, using the same notation.

simulation is much better, although it is still an underestimate. Apart from the lowest density, which is not far from the isotropic-nematic transition region, these higher-order virial theories all agree with the simulation results to within $\pm 10 \%$. However, as the order of the virial expansion goes up, the results begin to oscillate around the correct value. The third-order results are underestimates; the fourth-order results are slight overestimates, fifth order is a greater overestimate, while the sixth-order results are underestimates, barely distinguishable from the results obtained at third or- der. This is due to the appearance of negative coefficients in the theory at this level. Similar convergence properties are seen for $K_{2}$ and $K_{3}$, with second level virial theory significantly underestimating the elastic constant, and subsequent levels getting closer to the true value, until the change in sign of the virial coefficients causes fluctuation around the simulation result.

\section{CONCLUSIONS}

We have determined the elastic constants for the nematic liquid crystal phase of infinitesimally thin hard circular platelets. Comparison with the same quantities predicted by virial expansions shows that the lowest-level theory (second order) significantly underestimates the correct values, but that higher-order expansions are typically correct to within $10 \%$ at moderate to high density. However, the theory does not converge monotonically towards the correct result, but oscillates as the order increases, due to the appearance of highorder negative virial coefficients. Both theory and simulation show that the bend elastic constant is at least an order of magnitude smaller than the twist and splay constants. Finally, good agreement is obtained with experimental measurements of splay and bend elastic constants for a colloidal suspension of platelike particles.

\section{ACKNOWLEDGMENTS}

Computational resources were provided by the Centre for Scientific Computing, University of Warwick, and Manchester Computing, University of Manchester. EPSRC funded P.A.O. and M.D. Discussions with Henk Lekkerkerker and Tanja Schilling are gratefully acknowledged, and the authors of Ref. [24] are thanked for providing a preprint.
[1] P. G. de Gennes and J. Prost, The Physics of Liquid Crystals (Clarendon, Oxford, 1995).

[2] L. Onsager, Ann. N.Y. Acad. Sci. 51, 627 (1949).

[3] D. Frenkel and R. Eppenga, Phys. Rev. Lett. 49, 1089 (1982).

[4] R. Eppenga and D. Frenkel, Mol. Phys. 52, 1303 (1984).

[5] D. Frenkel, B. M. Mulder, and J. P. McTague, Phys. Rev. Lett. 52, 287 (1984).

[6] D. Frenkel and B. M. Mulder, Mol. Phys. 55, 1171 (1985).

[7] S. Fraden, in Observation, Prediction and Simulation of Phase Transitions in Complex Fluids, edited by M. Baus, L. F. Rull, and J.-P. Ryckaert (Kluwer Academic, Dordrecht, 1995), Vol. 460 of NATO ASI Series C, pp. 113-164.

[8] M. A. Bates and D. Frenkel, J. Chem. Phys. 110, 6553 (1999).

[9] F. M. van der Kooij, D. van der Beek, and H. N. W. Lekkerkerker, J. Phys. Chem. B 105, 1696 (2001).

[10] C. Oseen, Trans. Faraday Soc. 29, 883 (1933).

[11] F. C. Frank, Discuss. Faraday Soc. 25, 19 (1958).

[12] M. P. Allen and D. Frenkel, Phys. Rev. A 37, 1813 (1988).

[13] M. P. Allen and D. Frenkel, Phys. Rev. A 42, 3641(E) (1990).

[14] B. Tjipto-Margo, G. T. Evans, M. P. Allen, and D. Frenkel, J. Phys. Chem. 96, 3942 (1992).

[15] M. P. Allen, M. A. Warren, M. R. Wilson, A. Sauron, and W.
Smith, J. Chem. Phys. 105, 2850 (1996)

[16] G. T. Evans and E. B. Smith, Mol. Phys. 74, 79 (1991).

[17] B. Tjipto-Margo and G. T. Evans, J. Chem. Phys. 93, 4254 (1990).

[18] P. J. Camp, C. P. Mason, M. P. Allen, A. A. Khare, and D. A. Kofke, J. Chem. Phys. 105, 2837 (1996).

[19] D. Frenkel, J. Phys. Chem. 91, 4912 (1987).

[20] D. Frenkel, J. Phys. Chem. 92, 5314 (1988).

[21] J. Stelzer, M. A. Bates, L. Longa, and G. R. Luckhurst, J. Chem. Phys. 107, 7483 (1997).

[22] D. van der Beek, H. Reich, P. van der Schoot, M. Dijkstra, T. Schilling, R. Vink, M. Schmidt, R. van Roij, and H. N. W. Lekkerkerker, Phys. Rev. Lett. 97, 087801 (2006).

[23] D. van der Beek, P. Davidson, H. H. Wensink, G. J. Vroege, and H. N. W. Lekkerkerker, Phys. Rev. E 77, 031708 (2008).

[24] A. A. Verhoeff, R. H. J. Otten, P. van der Schoot, and H. N. W. Lekkerkerker, J. Phys. Chem. B (to be published).

[25] J. D. Parsons, Phys. Rev. A 19, 1225 (1979).

[26] S.-D. Lee, J. Chem. Phys. 87, 4972 (1987).

[27] F. H. Ree and W. G. Hoover, J. Chem. Phys. 40, 939 (1964).

[28] F. H. Ree and W. G. Hoover, J. Chem. Phys. 46, 4181 (1967).

[29] A. Poniewierski and J. Stecki, Mol. Phys. 38, 1931 (1979). 
[30] A. Poniewierski and J. Stecki, Phys. Rev. A 25, 2368 (1982).

[31] M. P. Allen, G. T. Evans, D. Frenkel, and B. Mulder, Adv. Chem. Phys. 86, 1 (1993).

[32] M. A. Bates, J. Chem. Phys. 111, 1732 (1999).
[33] D. Forster, Hydrodynamic Fluctuations, Broken Symmetry and Correlation Functions, Vol. 47 of Frontiers in Physics (Benjamin, Reading, 1975).

[34] M. Kroger and P. Ilg, J. Chem. Phys. 127, 034903 (2007). 